\title{
Temporal anterior encephalocele
}

\author{
Figure (A) Axial FLAIR, (B) coronal CT, (C) sagittal three-dimensional MR, and (D) fusion between CT and \\ MR showing the right temporal lobe herniating toward the pterygomaxillary fossa and the \\ sphenoidal sinus (arrows)
}

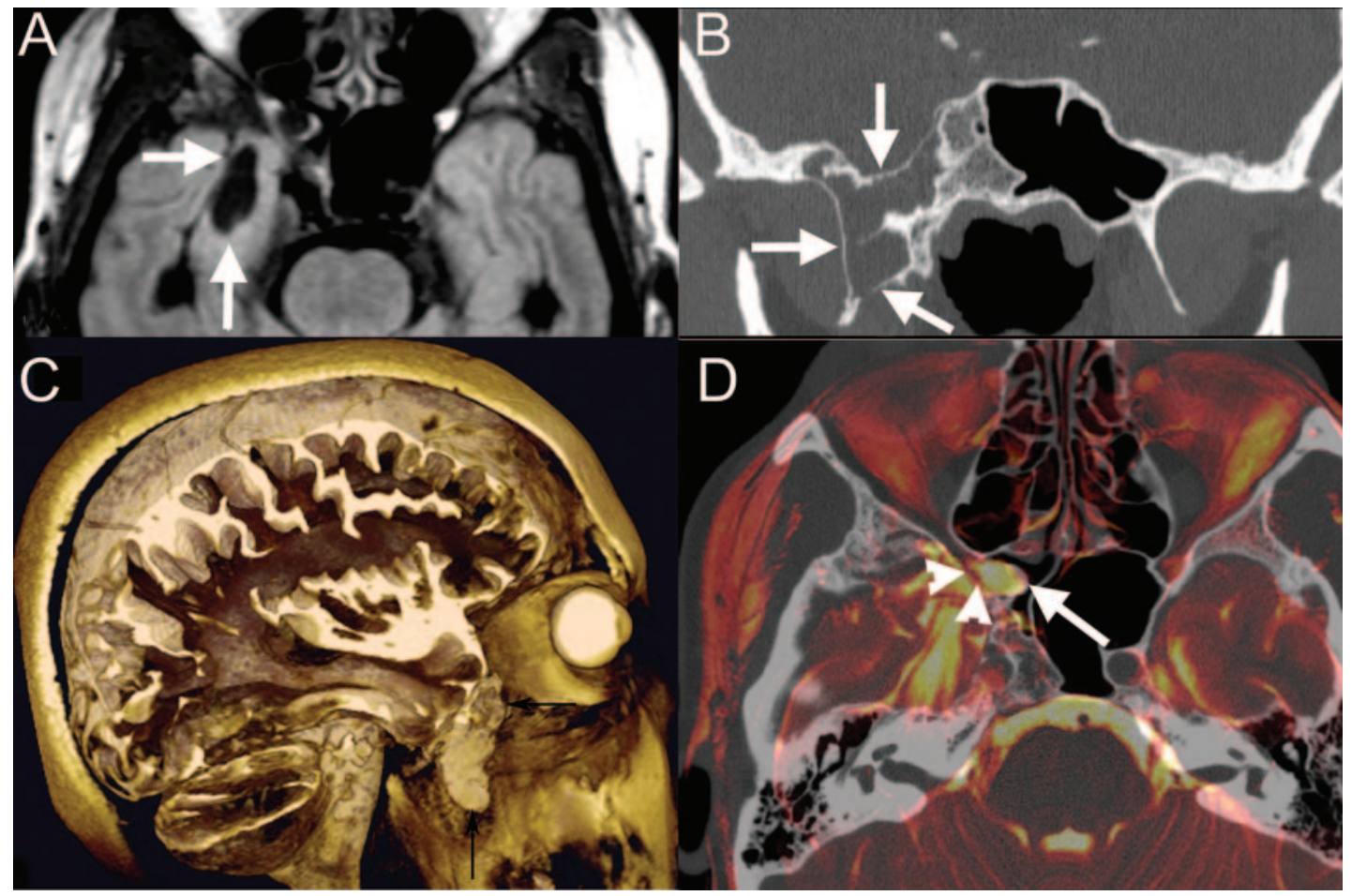

Fusion of images demonstrated the skull bone defect (arrowheads) and parenchyma in the sphenoidal sinus (arrows).

A 45-year-old woman had complex partial seizures for 25 years with pharmacoresistance for 2 years. EEG showed right temporal epileptiform discharges. MRI revealed atrophy of the right temporal parenchyma (figure, A) herniating toward the pterygomaxillary fossa and the sphenoidal sinus (figure, C). CT demonstrated the bone defect of the sphenoid wing (figure, B) whereas the fusion of both techniques identified this abnormality as an anterobasal temporal encephalocele (figure, D) causing this late-onset epilepsy. ${ }^{1,2}$

Encephaloceles can arise from neural tube defect or trauma. Such a congenital temporal encephalocele should be considered in the differential diagnosis of temporal epilepsy and should prompt a surgical workup which may be curative.

\section{M.I. Vargas, MD, S. Vulliemoz, MD, A. Rosset, MD, M. Seeck, MD, J. Delavelle, MD, Geneva, Switzerland}

Disclosure: The authors report no disclosures.

Address correspondence and reprint requests to Dr. Maria Isabel Vargas, Division of Neuroradiology, Department of Radiology, DISIM, Hôpital Universitaire HUG, 24 Micheli-du-Crest, 1211 Genève 14, Switzerland; maria.i.vargas@hcuge.ch

1. Leblanc R, Tampieri D, Robitaille Y, Olivier A, Andermann F, Sherwin A. Developmental anterobasal temporal encephalocele and temporal lobe epilepsy. J Neurosurg 1991;74:933-939.

2. Rojas L, Melvin JJ, Faerber EN, Valencia I. Anterior encephalocele associated with subependymal nodular heterotopia, cortical dysplasia and epilepsy: case report and review of the literature. Eur J Paediatr Neurol 2006;10:227-229. 


\section{Neurology}

Temporal anterior encephalocele

M. I. Vargas, S. Vulliemoz, A. Rosset, et al.

Neurology 2008;71;1293

DOI 10.1212/01.wnl.0000327602.65933.79

\section{This information is current as of October 13, 2008}

\section{Updated Information \& Services}

References

Citations

Subspecialty Collections

Permissions \& Licensing

Reprints including high resolution figures, can be found at: http://n.neurology.org/content/71/16/1293.full

This article cites 2 articles, 0 of which you can access for free at: http://n.neurology.org/content/71/16/1293.full\#ref-list-1

This article has been cited by 2 HighWire-hosted articles: http://n.neurology.org/content/71/16/1293.full\#\#otherarticles

This article, along with others on similar topics, appears in the following collection(s):

All Epilepsy/Seizures

http://n.neurology.org/cgi/collection/all_epilepsy_seizures

CT

http://n.neurology.org/cgi/collection/ct

Epilepsy semiology

http://n.neurology.org/cgi/collection/epilepsy_semiology

MRI

http://n.neurology.org/cgi/collection/mri

Volumetric MRI use in epilepsy

http://n.neurology.org/cgi/collection/volumetric_mri_use_in_epilepsy

Information about reproducing this article in parts (figures,tables) or in its entirety can be found online at:

http://www.neurology.org/about/about_the_journal\#permissions

Information about ordering reprints can be found online:

http://n.neurology.org/subscribers/advertise

Neurology ${ }^{\circledR}$ is the official journal of the American Academy of Neurology. Published continuously since 1951, it is now a weekly with 48 issues per year. Copyright . All rights reserved. Print ISSN: 0028-3878. Online ISSN: 1526-632X.

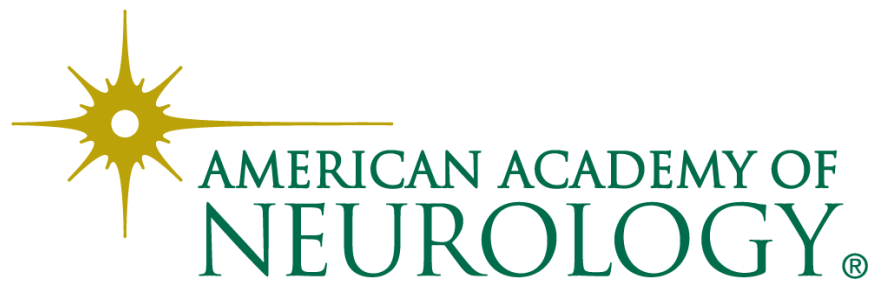

